Bull. Korean Math. Soc. 52 (2015), No. 4, pp. 1077-1095

http://dx.doi.org/10.4134/BKMS.2015.52.4.1077

\title{
EQUIVARIANT CROSSED MODULES AND COHOMOLOGY OF GROUPS WITH OPERATORS
}

\author{
Pham Thi Cuc and Nguyen Tien Quang
}

\begin{abstract}
In this paper we study equivariant crossed modules in its link with strict graded categorical groups. The resulting Schreier theory for equivariant group extensions of the type of an equivariant crossed module generalizes both the theory of group extensions of the type of a crossed module and the one of equivariant group extensions.
\end{abstract}

\section{Introduction}

Crossed modules and categorical groups have been used widely and independently, and in various contexts. In 1976 Brown and Spencer [3] showed that crossed modules are defined by $\mathcal{G}$-groupoids, and hence crossed modules can be studied by means of category theory. The notion of $\mathcal{G}$-groupoid is also called strict 2-group by Baez and Lauda [1], or strict categorical group by Joyal and Street [11].

A categorical group is a monoidal category in which every morphism is invertible and every object has a weak inverse. (Here, a weak inverse of an object $x$ is an object $y$ such that $x \otimes y$ and $y \otimes x$ are both isomorphic to the unit object.) A strict categorical group is a strict monoidal category in which every morphism is invertible and every object has a strict inverse (so that $x \otimes y$ and $y \otimes x$ are actually equal to the unit object).

Graded categorical groups were originally introduced by Fröhlich and Wall in [9]. Cegarra et al. [6] have proved a precise theorem on the homotopy classification of graded categorical groups and their homomorphisms thanks to the 3-dimensional equivariant cohomology group in the sense of [7]. Then, these results were applied to give an appropriate treatment of the equivariant group extensions with a non-abelian kernel in [6].

Brown and Mucuk classified group extensions of the type of a crossed module in [2]. Another generalized version of group extension is the equivariant group extension stated by Cegarra et al. thanks to the graded categorical group theory

Received July 30, 2013; Revised November 21, 2014.

2010 Mathematics Subject Classification. 18D10, 18D30, 20E22, 20 J06.

Key words and phrases. $\Gamma$-crossed module, strict graded categorical group, regular graded monoidal functor, equivariant extension, equivariant cohomology. 
(see $[6]$ ). One can recognize a generalization of both theories by means of $\Gamma$ crossed modules and strict graded categorical groups, which we deal with in this work.

The plan of this paper, briefly, is as follows. After this introductory Section 1 , Section 2 is devoted to recalling some fundamental results and notions of reduced graded categorical groups, the obstruction theory of a monoidal $\Gamma$ functor and a result on factor sets that will be used in the next section. In Section 3 we introduce the notion of strict graded categorical group, and show that any $\Gamma$-equivariant crossed module is defined by a strict graded categorical group. Then, we prove that the category ${ }_{\Gamma} \mathbf{G r s t r}$ of strict $\Gamma$-graded categorical groups and regular $\Gamma$-graded monoidal functors is equivalent to the category ${ }_{\Gamma}$ Cross of $\Gamma$-equivariant crossed modules (Theorem 7). A morphism in the category ${ }_{\Gamma}$ Cross consists of a homomorphism of $\Gamma$-equivariant crossed modules, $\left(f_{1}, f_{0}\right): \mathcal{M} \rightarrow \mathcal{M}^{\prime}$, and an element of the group of equivariant 2-cocycles $Z^{2}\left(\pi_{0} \mathcal{M}, \pi_{1} \mathcal{M}^{\prime}\right)$. This result contains a classical one, Theorem 1 [3].

Last, Section 4 is dedicated to stating Schreier theory for equivariant group extensions of the type of a $\Gamma$-crossed module by means of $\Gamma$-graded monoidal functors (Theorem 10, Theorem 12), then the classification theorem of group extensions of the type of a crossed module of Brown and Mucuk (Theorem $5.2[2])$ and that of $\Gamma$-group extensions of Cegarra et al. (Theorem 4.1 [6]) are obtained as particular cases.

\section{Reduced graded categorical groups}

Throughout $\Gamma$ is a fixed group. Let us recall that a $\Gamma$-group $\Pi$ means a group $\Pi$ enriched with a left $\Gamma$-action by automorphisms, and that a (left) $\Gamma$ equivariant $\Pi$-module is a $\Gamma$-module $A$, that is, an abelian $\Gamma$-group, endowed with a $\Pi$-module structure such that $\sigma(x a)=(\sigma x)(\sigma a)$ for all $\sigma \in \Gamma, x \in \Pi$ and $a \in A$. A $\Gamma$-homomorphism $f: \Pi \rightarrow \Pi^{\prime}$ of $\Gamma$-groups is a group homomorphism satisfying $f(\sigma x)=\sigma f(x), \sigma \in \Gamma, x \in \Pi$.

We regard the group $\Gamma$ as a category with one object, say $*$, where the morphisms are elements of $\Gamma$ and the composition is the group operation. A category $\mathbb{P}$ is $\Gamma$-graded if there is a functor $g r: \mathbb{P} \rightarrow \Gamma$. The grading $g r$ is said to be stable if for any object $X \in \mathrm{ObP}$ and any $\sigma \in \Gamma$ there exists an isomorphism $f$ in $\mathbb{P}$ with domain $X$ and $g r(f)=\sigma$. A $\Gamma$-graded monoidal category $\mathbb{P}=(\mathbb{P}$, gr, $\otimes, I, \mathbf{a}, \mathbf{l}, \mathbf{r})$ consists of:

1. a stable $\Gamma$-graded category $(\mathbb{P}$, gr $), \Gamma$-graded functors $\otimes: \mathbb{P} \times_{\Gamma} \mathbb{P} \rightarrow \mathbb{P}$ and $I: \Gamma \rightarrow \mathbb{P}$,

2. natural isomorphisms of grade $1 \mathbf{a}_{X, Y, Z}:(X \otimes Y) \otimes Z \stackrel{\sim}{\rightarrow} X \otimes(Y \otimes Z), \mathbf{l}_{X}$ : $I \otimes X \stackrel{\sim}{\rightarrow} X, \mathbf{r}_{X}: X \otimes I \stackrel{\sim}{\rightarrow} X$ such that, for all $X, Y, Z, T \in \mathrm{Ob} \mathbb{P}$, the following two coherence conditions hold:

$$
\begin{gathered}
\mathbf{a}_{X, Y, Z \otimes T} \mathbf{a}_{X \otimes Y, Z, T}=\left(i d_{X} \otimes \mathbf{a}_{Y, Z, T}\right) \mathbf{a}_{X, Y \otimes Z, T}\left(\mathbf{a}_{X, Y, Z} \otimes i d_{T}\right), \\
\left(i d_{X} \otimes \mathbf{l}_{Y}\right) \mathbf{a}_{X, I, Y}=\mathbf{r}_{X} \otimes i d_{Y} .
\end{gathered}
$$


A graded categorical group is a graded monoidal category $\mathbb{P}$ in which every object is invertible and every morphism is an isomorphism. In this case, the subcategory Ker $\mathbb{P}$ consisting of all objects of $\mathbb{P}$ and all morphisms of grade 1 in $\mathbb{P}$ is a categorical group.

If $\mathbb{P}, \mathbb{P}^{\prime}$ are $\Gamma$-monoidal categories, then a graded monoidal functor $(F, \widetilde{F}$, $\left.F_{*}\right): \mathbb{P} \rightarrow \mathbb{P}^{\prime}$ consists of a $\Gamma$-graded functor $F: \mathbb{P} \rightarrow \mathbb{P}^{\prime}$, natural isomorphisms of grade $1 \widetilde{F}_{X, Y}: F X \otimes F Y \rightarrow F(X \otimes Y)$, and an isomorphism of grade $1 F_{*}: I^{\prime} \rightarrow F I$, such that, for all $X, Y, Z \in \mathrm{ObP}$, the following coherence conditions hold:

$$
\begin{gathered}
\widetilde{F}_{X, Y \otimes Z}\left(i d_{F X} \otimes \widetilde{F}_{Y, Z}\right) \mathbf{a}_{F X, F Y, F Z}=F\left(\mathbf{a}_{X, Y, Z}\right) \widetilde{F}_{X \otimes Y, Z}\left(\widetilde{F}_{X, Y} \otimes i d_{F Z}\right), \\
F\left(\mathbf{r}_{X}\right) \widetilde{F}_{X, I}\left(i d_{F X} \otimes F_{*}\right)=\mathbf{r}_{F X}, F\left(\mathbf{l}_{X}\right) \widetilde{F}_{I, X}\left(F_{*} \otimes i d_{F X}\right)=\mathbf{l}_{F X} .
\end{gathered}
$$

Let $\left(F, \widetilde{F}, F_{*}\right),\left(F^{\prime}, \widetilde{F}^{\prime}, F_{*}^{\prime}\right)$ be two $\Gamma$-graded monoidal functors. A graded monoidal natural equivalence $\theta: F \stackrel{\sim}{\rightarrow} F^{\prime}$ is a natural equivalence of functors such that all isomorphisms $\theta_{X}: F X \rightarrow F^{\prime} X$ are of grade 1, and for all $X, Y \in \mathrm{Ob} \mathbb{P}$, the following coherence conditions hold:

$$
\widetilde{F}_{X, Y}^{\prime}\left(\theta_{X} \otimes \theta_{Y}\right)=\theta_{X \otimes Y} \widetilde{F}_{X, Y}, \theta_{I} F_{*}=F_{*}^{\prime} .
$$

The authors of $[6]$ showed that any $\Gamma$-graded categorical group $\mathbb{P}=(\mathbb{P}, g r, \otimes$, $I, \mathbf{a}, \mathbf{l}, \mathbf{r})$ determines a triple $(\Pi, A, h)$, where

- the set $\Pi=\pi_{0} \mathbb{P}$ of 1 -isomorphism classes of the objects in $\mathbb{P}$ is a $\Gamma$ group,

- the set $A=\pi_{1} \mathbb{P}$ of 1-automorphisms of the unit object $I$ is a $\Gamma$ equivariant $\Pi$-module,

- the third invariant is an equivariant cohomology class $\bar{h} \in H_{\Gamma}^{3}(\Pi, A)$ in the sense of [7].

Based on the data: a $\Gamma$-group $\Pi$, a $\Gamma$-equivariant $\Pi$-module $A$ and an element $h \in Z^{3}(\Pi, A)$, the $\Gamma$-graded categorical group $\int_{\Gamma}(\Pi, A, h)$ was constructed in [6] as follows. Objects of $\int_{\Gamma}(\Pi, A, h)$ are elements $x \in \Pi$ and their morphisms are pairs $(a, \sigma): x \rightarrow y$ consisting of an element $a \in A$ and $\sigma \in \Gamma$ such that $\sigma x=y$.

The composition $(x \stackrel{(a, \sigma)}{\longrightarrow} y \stackrel{(b, \tau)}{\longrightarrow} z)$ is given by

$$
(b, \tau)(a, \sigma)=(b+\tau a+h(x, \tau, \sigma), \tau \sigma) .
$$

The graded tensor product of two morphisms is given by

$$
(x \stackrel{(a, \sigma)}{\rightarrow} y) \otimes\left(x^{\prime} \stackrel{(b, \sigma)}{\rightarrow} y^{\prime}\right)=\left(x x^{\prime} \stackrel{\left(a+y b+h\left(x, x^{\prime}, \sigma\right), \sigma\right)}{\longrightarrow} y y^{\prime}\right) .
$$

The unit constrains are identities, $\mathbf{l}_{x}=(0,1)=\mathbf{r}_{x}: x \rightarrow x$. The associativity isomorphisms are

$$
\mathbf{a}_{x, y, z}=(h(x, y, z), 1):(x y) z \rightarrow x(y z) .
$$


The stable $\Gamma$-grading is $\operatorname{gr}(a, \sigma)=\sigma$. The unit graded functor $I: \Gamma \rightarrow$ $\int_{\Gamma}(\Pi, A, h)$ is given by

$$
I(* \stackrel{\sigma}{\rightarrow} *)=(1 \stackrel{(0, \sigma)}{\longrightarrow} 1) .
$$

In the case where $\Pi, A, \bar{h}$ are three invariants of a $\Gamma$-graded categorical group $\mathbb{P}$, then $\int_{\Gamma}(\Pi, A, h)$ is graded monoidally equivalent to $\mathbb{P}$. We call $\int_{\Gamma}(\Pi, A, h)$ a reduction of the $\Gamma$-graded categorical group $\mathbb{P}$, and simply denoted by $\mathcal{S}_{\mathbb{P}}$.

A $\Gamma$-functor $F: \int_{\Gamma}(\Pi, A, h) \rightarrow \int_{\Gamma}\left(\Pi^{\prime}, A^{\prime}, h^{\prime}\right)$ is of type $(\varphi, f)$ if

$$
F(x)=\varphi(x), \quad F(a, \sigma)=(f(a), \sigma), x \in \Pi, a \in A, \sigma \in \Gamma,
$$

where $\varphi: \Pi \rightarrow \Pi^{\prime}$ is a $\Gamma$-homomorphism (so that $A^{\prime}$ becomes a $\Gamma$-equivariant $\Pi$ module via $\varphi$ ) and $f: A \rightarrow A^{\prime}$ is a homomorphism of $\Gamma$-equivariant $\Pi$-modules (that is, a homomorphism which is both of $\Gamma$ - and $\Pi$-modules). In this case, we call $(\varphi, f)$ a pair of $\Gamma$-homomorphisms, and call the function

$$
\xi=\varphi^{*} h^{\prime}-f_{*} h
$$

an obstruction of the $\Gamma$-functor $F$, where $\varphi^{*}, f_{*}$ are canonical homomorphisms

$$
Z_{\Gamma}^{3}(\Pi, A) \stackrel{f_{*}}{\longrightarrow} Z_{\Gamma}^{3}\left(\Pi, A^{\prime}\right) \stackrel{\varphi^{*}}{\longleftarrow} Z_{\Gamma}^{3}\left(\Pi^{\prime}, A^{\prime}\right) .
$$

From Propositions 4 and $5[15]$ on the monoidal functors of type $(\varphi, f)$, one obtains the following proposition with some appropriate modifications.

Proposition 1. Let $\mathbb{P}$ and $\mathbb{P}^{\prime}$ be $\Gamma$-graded categorial groups, $\mathcal{S}_{\mathbb{P}}$ and $\mathcal{S}_{\mathbb{P}^{\prime}}$ be their reductions, respectively.

i) Every $\Gamma$-graded monoidal functor $(F, \widetilde{F}): \mathbb{P} \rightarrow \mathbb{P}^{\prime}$ induces one $S_{F}$ : $\mathcal{S}_{\mathbb{P}} \rightarrow \mathcal{S}_{\mathbb{P}^{\prime}}$.

ii) Every $\Gamma$-graded monoidal functor $\int_{\Gamma}(\Pi, A, h) \rightarrow \int_{\Gamma}\left(\Pi^{\prime}, A^{\prime}, h^{\prime}\right)$ is a $\Gamma$ functor of type $(\varphi, f)$.

The following proposition is a part of Theorem 3.2 [6].

Proposition 2. Let $\mathbb{P}, \mathbb{P}^{\prime}$ be two $\Gamma$-graded categorical groups. Any $\Gamma$-graded functor $(F, \widetilde{F}): \mathbb{P} \rightarrow \mathbb{P}^{\prime}$ induces a pair of $\Gamma$-homomorphisms $(\varphi, f)$, which realizes a $\Gamma$-graded monoidal functor, if and only if its obstruction $\bar{\xi}$ vanishes in $H_{\Gamma}^{3}\left(\pi_{0} \mathbb{P}, \pi_{1} \mathbb{P}^{\prime}\right)$. Then, there is a bijection

$$
\operatorname{Hom}_{(\varphi, f)}\left[\mathbb{P}, \mathbb{P}^{\prime}\right] \leftrightarrow H_{\Gamma}^{2}\left(\pi_{0} \mathbb{P}, \pi_{1} \mathbb{P}^{\prime}\right),
$$

where $\operatorname{Hom}_{(\varphi, f)}\left[\mathbb{P}, \mathbb{P}^{\prime}\right]$ is the set of all homotopy classes of monoidal $\Gamma$-functors from $\mathbb{P}$ to $\mathbb{P}^{\prime}$ inducing the pair of $\Gamma$-homomorphisms $(\varphi, f)$.

- Definition of factor sets with coefficients in a categorical group.

The notion of factor set in the Schreier-Eilenberg-Mac Lane theory for group extensions has been raised to categorical level by Grothendieck [10] and was also applied in [4], [5], [17]. In this paper we use this notion to define a strict graded categorical group. 
Definition. A factor set $\mathcal{F}$ on $\Gamma$ with coefficients in a categorical group $\mathbb{G}$ (or a pseudo-functor from $\Gamma$ to the category of categorical groups in the sense of Grothendieck [10]) consists of a family of monoidal autoequivalences $F^{\sigma}: \mathbb{G} \rightarrow$ $\mathbb{G}, \sigma \in \Gamma$, and isomorphisms between monoidal functors $\theta^{\sigma, \tau}: F^{\sigma} F^{\tau} \rightarrow F^{\sigma \tau}$, $\sigma, \tau \in \Gamma$, satisfying the conditions:

i) $F^{1}=i d_{\mathbb{G}}$,

ii) $\theta^{1, \sigma}=i d_{F^{\sigma}}=\theta^{\sigma, 1}, \sigma \in \Gamma$,

iii) $\theta^{\sigma \tau, \gamma} \circ \theta^{\sigma, \tau} F^{\gamma}=\theta^{\sigma, \tau \gamma} \circ F^{\sigma} \theta^{\tau, \gamma}$ for all $\sigma, \tau, \gamma \in \Gamma$.

We write $\mathcal{F}=\left(\mathbb{G}, F^{\sigma}, \theta^{\sigma, \tau}\right)$, or simply $(F, \theta)$.

The following lemma comes from an analogous result on graded monoidal categories [5] or a part of Theorem 1.2 [18]. We sketch the proof since we need some of its details.

Lemma 3. Each $\Gamma$-graded categorical group $(\mathbb{P}$, gr $)$ determines a factor set $\mathcal{F}$ on $\Gamma$ with coefficients in the categorical group $\operatorname{Ker} \mathbb{P}$.

Proof. For $\sigma \in \Gamma$, we define a monoidal autoequivalence $F^{\sigma}=\left(F^{\sigma}, \widetilde{F}^{\sigma}\right)$ : Ker $\mathbb{P} \rightarrow \operatorname{Ker} \mathbb{P}$ as follows: for each $X \in \operatorname{Ker} \mathbb{P}$, since the grading $g r$ is stable, there exists an isomorphism $\Upsilon_{X}^{\sigma}: X \stackrel{\sim}{\rightarrow} F^{\sigma} X$, where $F^{\sigma} X \in \operatorname{Ker} \mathbb{P}$, and $g r\left(\Upsilon_{X}^{\sigma}\right)=\sigma$. In particular, when $\sigma=1$ we take $F^{1} X=X$ and $\Upsilon_{X}^{1}=i d_{X}$. A morphism $f: X \rightarrow Y$ of grade 1 in $\mathrm{Ker} \mathbb{P}$ is carried to the unique morphism $F^{\sigma}(f)$ in $\operatorname{Ker} \mathbb{P}$ determined by

$$
F^{\sigma}(f)=\Upsilon_{Y}^{\sigma} \circ f \circ\left(\Upsilon_{X}^{\sigma}\right)^{-1} .
$$

The natural isomorphism $\widetilde{F}_{X, Y}^{\sigma}: F^{\sigma} X \otimes F^{\sigma} Y \stackrel{\sim}{\longrightarrow} F^{\sigma}(X \otimes Y)$ is determined by

$$
\widetilde{F}_{X, Y}^{\sigma}=\left(\Upsilon_{X}^{\sigma} \otimes \Upsilon_{Y}^{\sigma}\right) \circ\left(\Upsilon_{X \otimes Y}^{\sigma}\right)^{-1} .
$$

Furthermore, for each pair $\sigma, \tau \in \Gamma$ there is an isomorphism of monoidal functors $\theta^{\sigma, \tau}: F^{\sigma} F^{\tau} \stackrel{\sim}{\longrightarrow} F^{\sigma \tau}$, with $\theta^{1, \sigma}=i d_{F^{\sigma}}=\theta^{\sigma, 1}$, which is defined, for any $X \in \mathrm{Ob} \mathbb{P}$, by

$$
\theta_{X}^{\sigma, \tau}=\Upsilon_{F \tau}^{\sigma} \sigma \Upsilon_{X}^{\tau} \circ\left(\Upsilon_{X}^{\sigma \tau}\right)^{-1} .
$$

The pair $(F, \theta)$ constructed as above is a factor set.

\section{Strict graded categorial groups and $\Gamma$-crossed modules}

The objective of this paper is to extend the results on crossed modules and on equivariant group extensions. The notion of $\Gamma$-crossed module is a generalization of that of crossed module of groups of Whitehead [19]. First, observe that if $B$ is a $\Gamma$-group, then the group Aut $B$ of automorphisms of $B$ is also a $\Gamma$-group with the action

$$
(\sigma f)(b)=\sigma\left(f\left(\sigma^{-1} b\right)\right), b \in B, f \in \text { Aut } B .
$$

Then, the homomorphism $\mu: B \rightarrow$ Aut $B, b \mapsto \mu_{b}$ ( $\mu_{b}$ is the inner automorphism of $B$ given by conjugation with $b$ ) is a homomorphism of $\Gamma$-groups. 
Indeed, for all $\sigma \in \Gamma, a, b \in B$, one has

$$
\mu_{\sigma b}(a)=\sigma b+a-\sigma b=\sigma\left(b+\sigma^{-1} a-b\right)=\sigma\left(\mu_{b}\left(\sigma^{-1} a\right)\right)=\left(\sigma \mu_{b}\right)(a) .
$$

Definition. Let $B, D$ be $\Gamma$-groups. A $\Gamma$-crossed module is a quadruple $(B, D, d$, $\vartheta$ ), where $d: B \rightarrow D, \vartheta: D \rightarrow$ Aut $B$ are $\Gamma$-homomorphisms satisfying the following conditions:

$C_{1} \cdot \vartheta d=\mu$,

$C_{2} \cdot d\left(\vartheta_{x}(b)\right)=\mu_{x}(d(b))$,

$C_{3} \cdot \sigma\left(\vartheta_{x}(b)\right)=\vartheta_{\sigma x}(\sigma b)$,

where $\sigma \in \Gamma, x \in D, b \in B, \mu_{x}$ is the inner automorphism given by conjugation with $x$. $[12]$.

A $\Gamma$-crossed module is also called an $\Gamma$-equivariant crossed module by Noohi

Examples. Standard examples of $\Gamma$-crossed modules are:

1. $(B, D, i, \mu)$, where $i: B \rightarrow D$ is an inclusion $\Gamma$-homomorphism of a normal subgroup.

2. $(B, D, \mathbf{0}, \vartheta)$, where $B$ is a $D$-module, $\mathbf{0}: B \rightarrow D$ is the zero $\Gamma$-homomorphism, and $\vartheta$ is the module action.

3. $(B$, Aut $B, \mu, 0)$, where $\mu: B \rightarrow$ Aut $B$ is the $\Gamma$-homomorphism of any $\Gamma$-group $B$ which is given by conjugation.

4. $(B, D, p, \vartheta)$, where $p: B \rightarrow D$ is a $\Gamma$-surjective such that $\operatorname{Ker} p \subset Z(B)$, $\vartheta$ is given by conjugation.

Note on notations. For convenience, we denote by the addition for the operation in $B$ and by the multiplication for that in $D$. In this paper the $\Gamma$-crossed module $(B, D, d, \vartheta)$ is sometimes denoted by $B \stackrel{d}{\rightarrow} D$, or $B \rightarrow D$. In this section notations $\mathcal{M}, \mathcal{M}^{\prime}$ refer to the $\Gamma$-crossed modules $(B, D, d, \vartheta),\left(B^{\prime}, D^{\prime}, d^{\prime}, \vartheta^{\prime}\right)$, respectively.

The following properties follow from the definition of $\Gamma$-crossed module.

Proposition 4. Let $\mathcal{M}$ be a $\Gamma$-crossed module.

i) Kerd is a $\Gamma$-subgroup in $Z(B)$.

ii) Imd is both a normal subgroup in $D$ and a $\Gamma$-group.

iii) The $\Gamma$-homomorphism $\vartheta$ induces one $\varphi: D \rightarrow$ Aut(Kerd) by

$$
\varphi_{x}=\left.\vartheta_{x}\right|_{\text {Kerd }} \text {. }
$$

iv) Kerd is a left $\Gamma$-equivariant Cokerd-module under the actions

$$
s a=\varphi_{x}(a), \sigma s=[\sigma x], a \in \operatorname{Ker} d, x \in s \in \text { Cokerd } .
$$

The groups Kerd and Cokerd are denoted by $\pi_{1} \mathcal{M}$ and $\pi_{0} \mathcal{M}$, respectively.

It is well known that each crossed module of groups can be seen as a strict categorical group (see [3], [11, Remark 3.1]). Crossed modules of groups can be enriched in some ways to become, for example, crossed bimodules over rings, or equivariant crossed modules. In the former case, each crossed bimodule can be seen as a strict Ann-category [13]. In the later case, we shall show that each 
crossed module of $\Gamma$-groups can be identified with a strict $\Gamma$-graded categorical group. We now state this definition.

Recall that if $\left(F, \widetilde{F}, F_{*}\right)$ is a monoidal functor between categorical groups, then the isomorphism $F_{*}: I^{\prime} \rightarrow F I$ can be deduced from $F$ and $\widetilde{F}$, so we can omit $F_{*}$ when not necessary. A monoidal functor $(F, \widetilde{F}): \mathbb{G} \rightarrow \mathbb{G}^{\prime}$ between two categorical groups is termed regular if

$$
F(x) \otimes F(y)=F(x \otimes y), F(b) \otimes F(c)=F(b \otimes c)
$$

for all $x, y \in \mathrm{Ob} \mathbb{G}, b, c \in \operatorname{Mor} \mathbb{G}$. A factor set $(F, \theta)$ on $\Gamma$ with coefficients in a categorical group $\mathbb{G}$ is regular if $\theta^{\sigma, \tau}=i d$ and $F^{\sigma}$ is a regular monoidal functor, for all $\sigma \in \Gamma$.

Definition. A graded categorical group $(\mathbb{P}, g r)$ is said to be strict if

i) $\operatorname{Ker} \mathbb{P}$ is a strict categorical group,

ii) $\mathbb{P}$ induces a regular factor set $(F, \theta)$ on $\Gamma$ with coefficients in the categorical group Ker $\mathbb{P}$.

Equivalently, a graded categorical group $(\mathbb{P}, g r)$ is strict if it is a $\Gamma$-graded extension of a strict categorical group by a regular factor set.

For each $\Gamma$-crossed module $\mathcal{M}$, we construct a strict $\Gamma$-graded categorical group $\mathbb{P}_{\mathcal{M}}:=\mathbb{P}$ associated to $\mathcal{M}$.

Objects of $\mathbb{P}$ are elements of the group $D$, a $\sigma$-morphism $x \rightarrow y$ is a pair $(b, \sigma)$, where $b \in B, \sigma \in \Gamma$, such that $\sigma x=d(b) y$. The composition of two morphisms is defined by

$$
(x \stackrel{(b, \sigma)}{\rightarrow} y \stackrel{(c, \tau)}{\rightarrow} z)=(x \stackrel{(\tau b+c, \tau \sigma)}{\rightarrow} z) .
$$

This composition is associative and unitary since $B$ is a $\Gamma$-group.

For any morphism $(b, \sigma)$ in $\mathbb{P}$, one has

$$
(b, \sigma)^{-1}=\left(-\sigma^{-1} b, \sigma^{-1}\right),
$$

so that $\mathbb{P}$ is a groupoid. The tensor operation on objects is given by the multiplication in the group $D$, and for two morphisms $(x \stackrel{(b, \sigma)}{\rightarrow} y),\left(x^{\prime} \stackrel{(c, \sigma)}{\rightarrow} y^{\prime}\right)$, then

$$
(x \stackrel{(b, \sigma)}{\rightarrow} y) \otimes\left(x^{\prime} \stackrel{(c, \sigma)}{\longrightarrow} y^{\prime}\right)=\left(x x^{\prime} \stackrel{\left(b+\vartheta_{y} c, \sigma\right)}{\longrightarrow} y y^{\prime}\right),
$$

which is a functor thanks to the compatibility of the action $\vartheta$ with the $\Gamma$-action and conditions of the definition of $\Gamma$-crossed module, as below.

For morphisms $(x \stackrel{(b, \sigma)}{\rightarrow} y \stackrel{(c, \tau)}{\rightarrow} x),\left(x^{\prime} \stackrel{\left(b^{\prime}, \sigma\right)}{\rightarrow} y^{\prime} \stackrel{\left(c^{\prime}, \tau\right)}{\rightarrow} z^{\prime}\right)$ in $\mathbb{P}$

$$
\begin{aligned}
& (x \stackrel{(b, \sigma)}{\rightarrow} y \stackrel{(c, \tau)}{\longrightarrow} x) \otimes\left(x^{\prime} \stackrel{\left(b^{\prime}, \sigma\right)}{\longrightarrow} y^{\prime} \stackrel{\left(c^{\prime}, \tau\right)}{\longrightarrow} z^{\prime}\right) \\
\stackrel{(3)}{=} & (x \stackrel{(\tau b+c, \tau \sigma)}{\longrightarrow} z) \otimes\left(x^{\prime} \stackrel{\left(\tau b^{\prime}+c^{\prime}, \tau \sigma\right)}{\longrightarrow} z^{\prime}\right) \\
\stackrel{(4)}{=} & \left(x x^{\prime} \stackrel{\left(\tau b+c+\vartheta_{z}\left(\tau b^{\prime}+c^{\prime}\right), \tau \sigma\right)}{\longrightarrow} z z^{\prime}\right),
\end{aligned}
$$




$$
\begin{aligned}
& {\left[(x \stackrel{(b, \sigma)}{\longrightarrow} y) \otimes\left(x^{\prime} \stackrel{\left(b^{\prime}, \sigma\right)}{\longrightarrow} y^{\prime}\right)\right] \circ\left[(y \stackrel{(c, \tau)}{\longrightarrow} z) \otimes\left(y^{\prime} \stackrel{\left(c^{\prime}, \tau\right)}{\longrightarrow} z^{\prime}\right)\right] } \\
\stackrel{(4)}{=} & \left(x x^{\prime} \stackrel{\left(b+\vartheta_{y} b^{\prime}, \sigma\right)}{\longrightarrow} y y^{\prime}\right) \circ\left(y y^{\prime} \stackrel{\left(c+\vartheta_{z} c^{\prime}, \tau\right)}{\longrightarrow} z z^{\prime}\right) \\
\stackrel{(3)}{=} & \left(x x^{\prime} \stackrel{\left(\tau\left(b+\vartheta_{y} b^{\prime}\right)+c+\vartheta_{z} c^{\prime}, \tau \sigma\right)}{\longrightarrow} z z^{\prime}\right) .
\end{aligned}
$$

The fact that $\otimes$ is a functor is equivalent to

$$
\tau b+c+\vartheta_{z}\left(\tau b^{\prime}+c^{\prime}\right)=\tau\left(b+\vartheta_{y} b^{\prime}\right)+c+\vartheta_{z} c^{\prime} .
$$

This follows from

$$
\tau\left(\vartheta_{y} b^{\prime}\right) \stackrel{\left(C_{3}\right)}{=} \vartheta_{\tau y}\left(\tau b^{\prime}\right)=\vartheta_{(d c) z}\left(\tau b^{\prime}\right) \stackrel{\left(C_{1}\right)}{=} \mu_{c}\left(\vartheta_{z}\left(\tau b^{\prime}\right)\right)
$$

The associativity and unit constraints with respect to tensor product are strict. The graded functor is defined by $\operatorname{gr}(b, \sigma)=\sigma$, and the unit graded functor $I: \Gamma \rightarrow \mathbb{P}$ by

$$
I(* \stackrel{\sigma}{\rightarrow} *)=(1 \stackrel{(0, \sigma)}{\longrightarrow} 1) .
$$

Since $\mathrm{ObP}=D$ is a group and $x \otimes y=x y$, every object of $\mathbb{P}$ is invertible, whence $\operatorname{Ker} \mathbb{P}$ is a strict categorical group.

We next show that $\mathbb{P}$ induces a regular factor set $(F, \theta)$ on $\Gamma$ with coefficients in $\operatorname{KerP}$. For each $x \in D, \sigma \in \Gamma$, we set $F^{\sigma}(x)=\sigma x, \Upsilon_{x}^{\sigma}=(x \stackrel{(0, \sigma)}{\longrightarrow} \sigma x)$. Then, according to the proof of Lemma 3, we have $F^{\sigma}(b, 1)=(\sigma b, 1)$ and $\theta^{\sigma, \tau}=i d$. Now, it follows from the $\Gamma$-crossed module structure of $B \rightarrow D$ that $F^{\sigma}$ is a regular monoidal functor.

Thus, $\mathbb{P}$ is a strict $\Gamma$-graded categorical group.

Conversely, for each strict $\Gamma$-graded categorical group $\mathbb{P}$, we construct a $\Gamma$ crossed module associated to $\mathbb{P}$ as follows.

Set

$$
D=\mathrm{Ob} \mathbb{P}, \quad B=\{x \stackrel{b}{\rightarrow} 1 \mid x \in D, \operatorname{gr}(b)=1\} .
$$

The operations in $D$ and in $B$ are given by

$$
x y=x \otimes y, \quad b+c=b \otimes c,
$$

respectively. Then, $D$ becomes a group in which the unit is 1 , the inverse of $x$ is $x^{-1}\left(x \otimes x^{-1}=1\right)$. $B$ is a group in which the zero is the morphism $\left(1 \stackrel{i d_{1}}{\longrightarrow} 1\right)$ and the negative of $(x \stackrel{b}{\rightarrow} 1)$ is the morphism $\left(x^{-1} \stackrel{\bar{b}}{\rightarrow} 1\right)\left(b \otimes \bar{b}=i d_{1}\right)$.

By the definition of $\mathbb{P}$, its kernel KerP is a strict categorical group and $\mathbb{P}$ has a regular factor set $(F, \theta)$. Thus, $D, B$ are $\Gamma$-groups in which the actions are respectively defined by

$$
\begin{gathered}
\sigma x=F^{\sigma}(x), x \in D, \sigma \in \Gamma, \\
\sigma b=F^{\sigma}(b), b \in B .
\end{gathered}
$$

The correspondences $d: B \rightarrow D$ and $\vartheta: D \rightarrow$ Aut $B$ are given by

$$
d(x \stackrel{b}{\rightarrow} 1)=x
$$




$$
\vartheta_{y}(x \stackrel{b}{\rightarrow} 1)=\left(y x y^{-1} \stackrel{i d_{y}+b+i d_{y^{-1}}}{\longrightarrow} 1\right),
$$

respectively. Since $B, D$ are $\Gamma$-groups, it is easy to see that $d, \vartheta$ are $\Gamma$-homomorphisms.

Definition. A homomorphism $\left(f_{1}, f_{0}\right): \mathcal{M} \rightarrow \mathcal{M}^{\prime}$ of $\Gamma$-crossed modules consists of $\Gamma$-homomorphisms $f_{1}: B \rightarrow B^{\prime}, f_{0}: D \rightarrow D^{\prime}$ satisfying

$H_{1} \cdot f_{0} d=d^{\prime} f_{1}$,

$H_{2} . f_{1}\left(\vartheta_{x} b\right)=\vartheta_{f_{0}(x)}^{\prime} f_{1}(b)$ for all $x \in D, b \in B$.

The following lemmas state the relation between homomorphisms of $\Gamma$ crossed modules and graded monoidal functors between corresponding associated graded categorical groups. Observe that a morphism $(x \stackrel{(b, \sigma)}{\longrightarrow} y)$ in $\mathbb{P}_{B \rightarrow D}$ can be written as

$$
x \stackrel{(0, \sigma)}{\longrightarrow} \sigma x \stackrel{(b, 1)}{\longrightarrow} y,
$$

and a $\Gamma$-graded monoidal functor $(F, \widetilde{F}): \mathbb{P}_{\mathcal{M}} \rightarrow \mathbb{P}_{\mathcal{M}^{\prime}}$ defines a function $f$ : $D^{2} \cup D \times \Gamma \rightarrow B$ by

$$
(f(x, y), 1)=\widetilde{F}_{x, y}, \quad(f(x, \sigma), \sigma)=F(x \stackrel{(0, \sigma)}{\rightarrow} \sigma x)
$$

Lemma 5. Let $\left(f_{1}, f_{0}\right): \mathcal{M} \rightarrow \mathcal{M}^{\prime}$ be a homomorphism of $\Gamma$-crossed modules. Then, there exists a $\Gamma$-graded monoidal functor $(F, \widetilde{F}): \mathbb{P}_{\mathcal{M}} \rightarrow \mathbb{P}_{\mathcal{M}^{\prime}}$ defined by $F(x)=f_{0}(x), F(b, 1)=\left(f_{1}(b), 1\right)$, if and only if $f=p^{*} \varphi$, where $\varphi \in$ $Z_{\Gamma}^{2}\left(\right.$ Coker $d$, Ker $\left.d^{\prime}\right), p: D \rightarrow$ Coker $d$ is a canonical projection.

Proof. Since $f_{0}$ is a homomorphism and $F x=f_{0}(x), \widetilde{F}_{x, y}: F x F y \rightarrow F(x y)$ is a morphism of grade 1 in $\mathbb{P}^{\prime}$ if and only $d f(x, y)=1^{\prime}$, or $f(x, y) \in \operatorname{Ker} d^{\prime} \subset Z\left(B^{\prime}\right)$.

Also, since $f_{0}$ is a $\Gamma$-homomorphism, $(F x \stackrel{(f(x, \sigma), \sigma)}{\longrightarrow} F \sigma x)$ is a morphism of grade $\sigma$ in $\mathbb{P}^{\prime}$ if and only if $d f(x, \sigma)=1^{\prime}$, or $f(x, \sigma) \in \operatorname{Ker} d^{\prime} \subset Z\left(B^{\prime}\right)$. In particular, when $\sigma=1$ then $f\left(x, 1_{\Gamma}\right)=f_{1}(0)=0$.

The fact that $f_{1}$ is a group homomorphism is equivalent to the condition of $F$ preserving composition of morphisms of grade 1 . The condition of $F$ preserving the composition of morphisms of form $(0, \sigma)$ is equivalent to

$$
\tau f(x, \sigma)+f(\sigma x, \tau)=f(x, \tau \sigma) .
$$

- The condition of $\widetilde{F}_{x, y}$ being natural isomorphisms.

- For morphisms of grade 1, consider the diagram

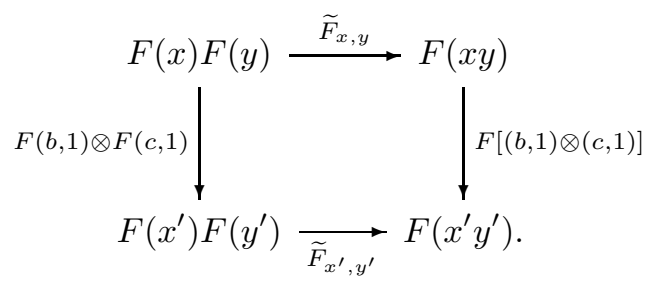


Since the homomorphisms $f_{0}, f_{1}$ satisfy the condition $\mathrm{H}_{2}$, the following equation holds:

$$
F(b, 1) \otimes F(c, 1)=F[(b, 1) \otimes(c, 1)] .
$$

Then, since $f(x, y), f\left(x^{\prime}, y^{\prime}\right) \in Z\left(B^{\prime}\right)$, the above diagram commutes if and only if

$$
f(x, y)=f\left(x^{\prime}, y^{\prime}\right)
$$

So, $\widetilde{F}$ defines a function $\varphi: \operatorname{Coker}^{2} d \rightarrow \operatorname{Ker} d^{\prime}$,

$$
\varphi(r, s)=f(x, y), r=p x, s=p y,
$$

where $p: D \rightarrow$ Coker $d$ is a canonical projection.

- For morphisms of form $(0, \sigma)$, the diagram

commutes if and only if

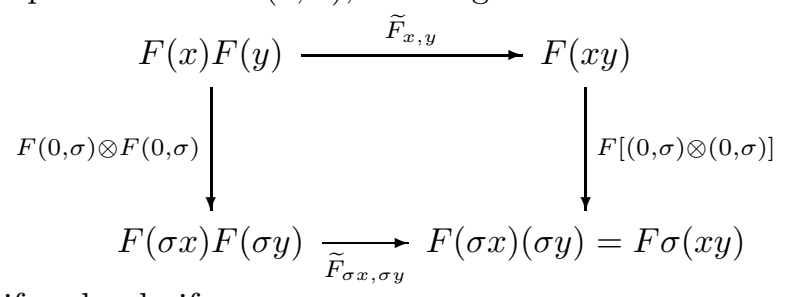

$$
\sigma f(x, y)-f(\sigma x, \sigma y)=f(x, \sigma)+\vartheta_{F(\sigma x)}^{\prime} f(y, \sigma)-f(x y, \sigma),
$$

or

$$
\sigma f(x, y)-f(\sigma x, \sigma y)=f(x, \sigma)+(\sigma x) f(y, \sigma)-f(x y, \sigma) .
$$

- The commutativity of diagram

leads to

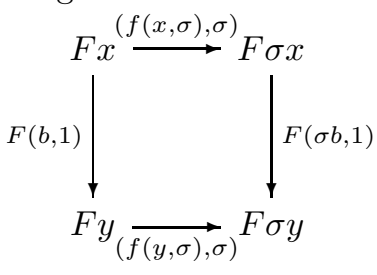

$$
f(x, \sigma)+f_{1}(\sigma b)=\sigma f_{1}(b)+f(y, \sigma) .
$$

Since $f_{1}$ is a $\Gamma$-homomorphism, it follows that $f(x, \sigma)=f(y, \sigma)$. This gives a function $\varphi$ : Coker $d \times \Gamma \rightarrow \operatorname{Ker} d^{\prime}$

$$
\varphi(r, \sigma)=f(x, \sigma), r=p x .
$$

Therefore, one obtains a function

$$
\varphi: \text { Coker }^{2} d \cup \text { Coker } d \times \Gamma \rightarrow \text { Ker } d^{\prime}
$$

which is normalized in the sense that

$$
\varphi(1, s)=\varphi(r, 1)=0=\varphi\left(r, 1_{\Gamma}\right) .
$$

The first two equations hold since $F(1)=1^{\prime}$ and $(F, \widetilde{F})$ is compatible with the unit constraints. The last one holds since $f\left(x, 1_{\Gamma}\right)=0$. 
The compatibility of $(F, \widetilde{F})$ with the associativity constraints implies

$$
\vartheta_{F x}^{\prime}(f(y, z))+f(x, y z)=f(x, y)+f(x y, z) .
$$

It follows from the relations (6)-(8) that $\varphi \in Z_{\Gamma}^{2}\left(\right.$ Coker $\left.d, \operatorname{Ker} d^{\prime}\right)$.

Note that the strict $\Gamma$-graded categorical group $\mathbb{P}$ induces a $\Gamma$-action on the group $D$ of objects and on the group $B$ of morphisms of grade 1 , we state the following definition.

Definition. A $\Gamma$-graded monoidal functor $(F, \widetilde{F}): \mathbb{P} \rightarrow \mathbb{P}^{\prime}$ between two strict $\Gamma$-graded categorical groups is called regular if:

$S_{1} . F(x \otimes y)=F(x) \otimes F(y)$,

$S_{2} . F(\sigma x)=\sigma F(x)$,

$S_{3} . F(\sigma b)=\sigma F(b)$,

$S_{4} . F(b) \otimes F(c)=F(b \otimes c)$

for $x, y \in \mathrm{Ob} \mathbb{P}$, and $b, c$ are morphisms of grade 1 in $\mathbb{P}$.

The $\Gamma$-graded monoidal functor mentioned in Lemma 5 is regular.

Thanks to Lemma 5, one can define the category ${ }_{\Gamma}$ Cross whose objects are $\Gamma$-crossed modules and whose morphisms are triples $\left(f_{1}, f_{0}, \varphi\right)$, where $\left(f_{1}, f_{0}\right)$ : $\mathcal{M} \rightarrow \mathcal{M}^{\prime}$ is a homomorphism of $\Gamma$-crossed modules and $\varphi \in Z_{\Gamma}^{2}\left(\operatorname{Coker} d\right.$, Ker $\left.d^{\prime}\right)$. The composition with the morphism $\left(f_{1}^{\prime}, f_{0}^{\prime}, \varphi^{\prime}\right): \mathcal{M}^{\prime} \rightarrow \mathcal{M}^{\prime \prime}$ is given by

$$
\left(f_{1}^{\prime}, f_{0}^{\prime}, \varphi^{\prime}\right) \circ\left(f_{1}, f_{0}, \varphi\right)=\left(f_{1}^{\prime} f_{1}, f_{0}^{\prime} f_{0},\left(f_{1}^{\prime}\right)_{*} \varphi+\left(f_{0}\right)^{*}\left(\varphi^{\prime}\right)\right) .
$$

Lemma 6. Let $\mathbb{P}$ and $\mathbb{P}^{\prime}$ be corresponding strict $\Gamma$-graded categorical groups associated to $\Gamma$-crossed modules $\mathcal{M}$ and $\mathcal{M}^{\prime}$, and let $(F, \widetilde{F}): \mathbb{P} \rightarrow \mathbb{P}^{\prime}$ be a regular $\Gamma$-graded monoidal functor. Then, the triple $\left(f_{1}, f_{0}, \varphi\right)$, where

i) $f_{0}(x)=F(x),\left(f_{1}(b), 1\right)=F(b, 1), \sigma \in \Gamma, b \in B, x, y \in D$,

ii) $p^{*} \varphi=f$ for $f$ is given by $(5)$,

is a morphism in the category ${ }_{\Gamma}$ Cross.

Proof. By the condition $S_{1}, f_{0}$ is a group homomorphism, and by the condition $S_{2}, f_{0}$ is a $\Gamma$-homomorphism. Since $F$ preserves the composition of morphisms of grade $1, f_{1}$ is a group homomorphism. Moreover, $f_{1}$ is a $\Gamma$-homomorphism thanks to the condition $S_{3}$. Each element $b \in B$ can be seen as a morphism $(d b \stackrel{(b, 1)}{\longrightarrow} 1)$ in $\mathbb{P}$, and hence $\left(f_{0}(d b) \stackrel{\left(f_{1}(b), 1\right)}{\longrightarrow} 1^{\prime}\right)$ is a morphism in $\mathbb{P}^{\prime}$, that means $H_{1}$ holds:

$$
f_{0}(d b)=d^{\prime}\left(f_{1}(b)\right) .
$$

By the condition $S_{4}$ and the fact that $f_{1}$ is a homomorphism, $H_{2}$ is satisfied:

$$
f_{1}\left(\vartheta_{y} c\right)=\vartheta_{f_{0}(y)}^{\prime} f_{1}(c) \text {. }
$$

Thus, $\left(f_{1}, f_{0}\right)$ is a homomorphism of $\Gamma$-crossed modules. By Lemma 5 , the function $f$ determines a function $\varphi \in Z_{\Gamma}^{2}\left(\operatorname{Coker} d\right.$, Ker $\left.d^{\prime}\right)$, where $f=p^{*} \varphi, p$ : $D \rightarrow$ Coker $d$ is the canonical projection. Therefore, $\left(f_{1}, f_{0}, \varphi\right)$ is a morphism in ${ }_{\Gamma}$ Cross. 
Denote by

\section{${ }_{\Gamma}$ Grstr}

the category of strict $\Gamma$-graded categorical groups and regular $\Gamma$-graded monoidal functors, we have the following result.

Theorem 7 (Classification Theorem). There exists an equivalence

$$
\begin{array}{clc}
\Phi:{ }_{\Gamma} \text { Cross } & \rightarrow & { }_{\Gamma} \text { Grstr } \\
(B \rightarrow D) & \mapsto & \mathbb{P}_{B \rightarrow D} \\
\left(f_{1}, f_{0}, \varphi\right) & \mapsto & (F, \widetilde{F})
\end{array}
$$

where $F(x)=f_{0}(x), F(b, 1)=\left(f_{1}(b), 1\right)$, and

$$
F(x \stackrel{(0, \sigma)}{\rightarrow} \sigma x)=(\varphi(p x, \sigma), \sigma), \widetilde{F}_{x, y}=(\varphi(p x, p y), 1)
$$

for $x, y \in D, b \in B, \sigma \in \Gamma$.

Proof. Let $\mathbb{P}, \mathbb{P}^{\prime}$ be the $\Gamma$-graded categorical groups associated to $\Gamma$-crossed modules $\mathcal{M}, \mathcal{M}^{\prime}$, respectively. By Lemma 5 , the correspondence $\left(f_{1}, f_{0}, \varphi\right) \mapsto$ $(F, \widetilde{F})$ defines an injection on the homsets,

$$
\Phi: \operatorname{Hom}_{\Gamma} \operatorname{Cross}\left(\mathcal{M}, \mathcal{M}^{\prime}\right) \rightarrow \operatorname{Hom}_{\Gamma} \operatorname{Grstr}\left(\mathbb{P}_{\mathcal{M}}, \mathbb{P}_{\mathcal{M}^{\prime}}\right) .
$$

By Lemma 6, $\Phi$ is surjective.

If $\mathbb{P}$ is a strict $\Gamma$-graded categorical group and $\mathcal{M}_{\mathbb{P}}$ is its associated $\Gamma$-crossed module, then $\Phi\left(\mathcal{M}_{\mathbb{P}}\right)=\mathbb{P}$ (rather than an isomorphism). Thus, $\Phi$ is an equivalence.

When $\Gamma=\mathbf{1}$, the trivial group, then the category ${ }_{1}$ Cross $=$ Cross has crossed modules as objects, the morphisms are triples $\left(f_{1}, f_{0}, \varphi\right)$, where $\left(f_{1}, f_{0}\right)$ : $\mathcal{M} \rightarrow \mathcal{M}^{\prime}$ is a homomorphism of crossed modules and $\varphi \in Z^{2}\left(\right.$ Cokerd, Kerd $\left.d^{\prime}\right)$, and the category ${ }_{\mathbf{1}} \mathbf{G r s t r}=\mathbf{G r s t r}$ has strict categorical groups as objects, the morphisms are regular monoidal functor. Thus, one obtains the following result.

Corollary 8 (Theorem $5[14]$ ). There exists an equivalence

$$
\begin{array}{rll}
\text { Cross } & \rightarrow & \text { Grstr } \\
(B \rightarrow D) & \mapsto & \mathbb{P}_{B \rightarrow D} \\
\left(f_{1}, f_{0}, \varphi\right) & \mapsto & (F, \widetilde{F})
\end{array}
$$

where $F(x)=f_{0}(x), F(b)=f_{1}(b), \widetilde{F}_{x, y}=\varphi(\bar{x}, \bar{y})$ for $x, y \in D, b \in B$.

The two categories mentioned in Corollary 8 have two corresponding subcategories:

- The category 2-Gp of $\mathcal{G}$-groupoids (by Brown and Spencer [3]) is a subcategory of the category Grstr in which the morphisms consist of monoidal functors $(F, \widetilde{F})$ with $\widetilde{F}=i d$.

- The category CrossMd of crossed modules (in the sense of usual) is the subcategory of the category Cross in which the morphisms consist of triples $\left(f_{1}, f_{0}, \varphi\right)$ with $\varphi=0$. 
The categories 2-Gp and CrossMd are equivalent via a functor which is a restriction of $\Phi$. Thus, Corollary 8 contains Theorem 1 [3].

\section{Equivariant group extensions, $\Gamma$-crossed modules and equivariant group cohomology}

In this section we develop a theory of equivariant group extensions of the type of a $\Gamma$-crossed module which extends both group extension theory of the type of a crossed module $[2,8,16]$ and equivariant group extension theory [6].

Definition. Let $B \stackrel{d}{\rightarrow} D$ be a $\Gamma$-crossed module and $Q$ be a $\Gamma$-group. An equivariant group extension of $B$ by $Q$ of type $B \stackrel{d}{\rightarrow} D$ is a diagram of $\Gamma$ homomorphisms

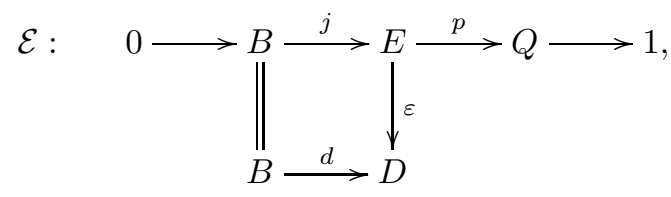

where the top row is exact, the family $\left(B, E, j, \vartheta^{0}\right)$ is a $\Gamma$-crossed module in which $\vartheta^{0}$ is given by conjugation, and $(i d, \varepsilon)$ is a homomorphism of $\Gamma$-crossed modules.

Two equivariant extensions of $B$ by $Q$ of type $B \stackrel{d}{\rightarrow} D$ are said to be equivalent if there is a morphism of exact sequences

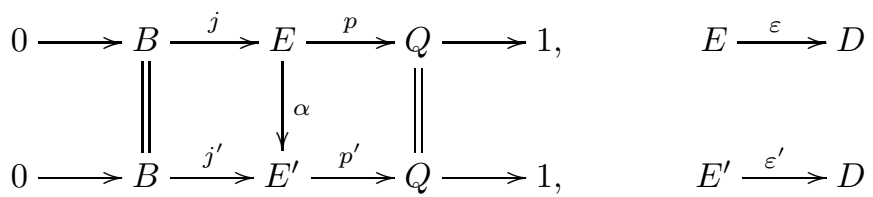

such that $\varepsilon^{\prime} \alpha=\varepsilon$. Obviously, $\alpha$ is a $\Gamma$-isomorphism.

In the diagram

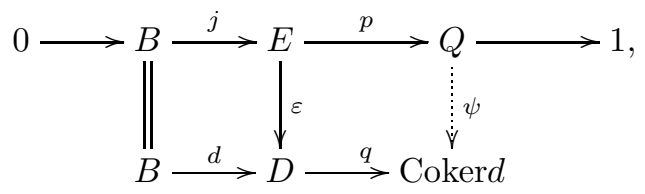

where $q$ is a canonical $\Gamma$-homomorphism, since the top row is exact and $q \circ \varepsilon \circ j=$ $q \circ d=0$, there is a $\Gamma$-homomorphism $\psi: Q \rightarrow$ Cokerd such that the right hand side square commutes. Moreover, $\psi$ is dependent only on the equivalence class of the extension $\mathcal{E}$, and we say that the extension $\mathcal{E}$ induces $\psi$. The set of equivalence classes of equivariant extensions of $B$ by $Q$ of type $B \rightarrow D$ inducing $\psi: Q \rightarrow$ Cokerd is denoted by

$$
\operatorname{Ext}_{B \rightarrow D}^{\Gamma}(Q, B, \psi) .
$$


Now, in order to study this set we apply the obstruction theory to $\Gamma$-graded monoidal functors between strict $\Gamma$-graded categorical groups ${ }_{\Gamma} \operatorname{Dis} Q$ and $\mathbb{P}_{B \rightarrow D}$, where the discrete $\Gamma$-graded categorical group ${ }_{\Gamma} \operatorname{Dis} Q$ is defined by

$$
{ }_{\Gamma} \operatorname{Dis} Q=\int_{\Gamma}(Q, 0,0) \text {. }
$$

This is just the strict $\Gamma$-graded categorical group associated to the $\Gamma$-crossed module $(0, Q, 0,0)$ (see Section 3). Thus, the objects of ${ }_{\Gamma} \operatorname{Dis} Q$ are the elements of $Q$ and its morphisms $\sigma: x \rightarrow y$ are the elements $\sigma \in \Gamma$ with $\sigma x=y$. Composition of morphisms is multiplication in $\Gamma$. The graded tensor product is given by

$$
(x \stackrel{\sigma}{\rightarrow} y) \otimes\left(x^{\prime} \stackrel{\sigma}{\rightarrow} y^{\prime}\right)=\left(x x^{\prime} \stackrel{\sigma}{\rightarrow} y y^{\prime}\right) .
$$

We first prove the following lemma.

Lemma 9. Let $B \rightarrow D$ be a $\Gamma$-crossed module, and let $\psi: Q \rightarrow$ Coker $d$ be a $\Gamma$-homomorphism. For each $\Gamma$-graded monoidal functor $(F, \widetilde{F}): \Gamma \operatorname{Dis} Q \rightarrow$ $\mathbb{P}_{B \rightarrow D}$ which satisfies $F(1)=1$ and induces a pair of $\Gamma$-homomorphisms $(\psi, 0)$ : $(Q, 0) \rightarrow($ Coker $d$, Ker $d)$, there exists an equivariant group extension $\mathcal{E}_{F}$ of $B$ by $Q$ of type $B \rightarrow D$ inducing $\psi$.

The extension $\mathcal{E}_{F}$ is called an equivariant crossed product extension associated to the $\Gamma$-graded monoidal functor $F$.

Proof. Let $(F, \widetilde{F}):{ }_{\Gamma}$ Dis $Q \rightarrow \mathbb{P}$ be a $\Gamma$-graded monoidal functor. By (5), it defines a function $f: Q \times Q \cup(Q \times \Gamma) \rightarrow B$ which is normalized in the sense that

$$
f\left(x, 1_{\Gamma}\right)=0=f(x, 1)=f(1, y) .
$$

The first equality holds since $F$ preserves identities, the rest ones hold since $F(1)=1$ and $F$ is compatible with the unit constraints.

It follows from the definition of morphism in $\mathbb{P}$ that

$$
\begin{gathered}
\sigma F(x)=d f(x, \sigma) F(\sigma x), \\
F(x) F(y)=d f(x, y) F(x y) .
\end{gathered}
$$

According to the proof of Lemma 5 , the function $f$ satisfies the equations (6)(8), but it here takes values in $B$ instead of Ker $d^{\prime}$.

Firstly, we construct the equivariant crossed product $E_{0}=B \times_{f} Q$. The $\Gamma$-group structure of $E_{0}$ is given by the rules

$$
\begin{aligned}
(b, x)+(c, y) & =\left(b+\vartheta_{F x}(c)+f(x, y), x y\right), \\
\sigma(b, x) & =(\sigma b+f(x, \sigma), \sigma x) .
\end{aligned}
$$

Thanks to the conditions (6), (10) and (12), $B \times_{f} Q$ is actually a group. The zero is $(0,1)$ and $-(b, x)=\left(b^{\prime}, x^{-1}\right)$, where $\vartheta_{F x}\left(b^{\prime}\right)=-b-f\left(x, x^{-1}\right)$. Moreover, $E_{0}$ is a $\Gamma$-group owning to the conditions (7), (8) and (11). 
Then, the following sequence is exact

$$
\mathcal{E}_{F}: \quad 0 \rightarrow B \stackrel{j_{0}}{\rightarrow} E_{0} \stackrel{p_{0}}{\rightarrow} Q \rightarrow 1,
$$

where $j_{0}(b)=(b, 1), p_{0}(b, x)=x, b \in B, x \in Q$. Since $j_{0}(B)$ is a normal subgroup in $E_{0}, j_{0}: B \rightarrow E_{0}$ is a $\Gamma$-crossed module in which the action $\vartheta^{0}$ : $E_{0} \rightarrow$ Aut $B$ is given by conjugation.

Next, we embed $\mathcal{E}_{F}$ into the diagram $(9)$ as follows. Since $(F, \widetilde{F})$ induces a $\Gamma$-homomorphism $\psi: Q \rightarrow$ Coker $d$ by $\psi(x)=[F x] \in$ Coker $d$, the elements $F x$ are representatives of Cokerd in $D$. Then, the map $\varepsilon: E_{0} \rightarrow D$ given by

$$
\varepsilon(b, x)=d b . F x
$$

is a $\Gamma$-homomorphism thanks to the conditions (11) and (12).

It is easy to see that $\varepsilon \circ j_{0}=d$. Besides, for all $(b, x) \in E_{0}, c \in B$, one has $\vartheta_{(b, x)}^{0}(c)=\vartheta_{\varepsilon(b, x)}(c)$, as calculated below:

$$
\begin{gathered}
\vartheta_{(b, x)}^{0}(c)=j_{0}^{-1}\left[\mu_{(b, x)}(c, 1)\right]=\mu_{b}\left[\vartheta_{F x}(c)\right], \\
\vartheta_{\varepsilon(b, x)}(c)=\vartheta_{d b . F x}(c)=\mu_{b}\left[\vartheta_{F x}(c)\right] .
\end{gathered}
$$

Finally, for all $x \in Q$,

$$
q \varepsilon(b, x)=q(d b . F x)=q(F x)=\psi(x)=\psi p_{0}(b, x),
$$

so that the extension $\mathcal{E}_{F}$ induces the $\Gamma$-homomorphism $\psi: Q \rightarrow$ Coker $d$.

Under the hypothesis of Lemma 9, we state the following theorem.

Theorem 10 (Schreier theory for equivariant group extensions of the type of a $\Gamma$-crossed module). There is a bijection

$$
\operatorname{Ext}_{B \rightarrow D}^{\Gamma}(Q, B, \psi) \leftrightarrow \operatorname{Hom}_{(\psi, 0)}\left[\operatorname{Dis}_{\Gamma} Q, \mathbb{P}_{B \rightarrow D}\right]
$$

Proof. First, every $\Gamma$-graded monoidal functor $(F, \widetilde{F})$ is homotopic to one $(G, \widetilde{G})$ in which $G(1)=1$. Hence, we can restrict our attention to this kind of $\Gamma$-graded monoidal functor $(F, \widetilde{F}): \operatorname{Dis}_{\Gamma} Q \rightarrow \mathbb{P}_{B \rightarrow D}$. The, by Lemma 9, each $\Gamma$-graded monoidal functor $(F, \widetilde{F})$ defines an equivariant extension $\mathcal{E}_{F} \in$ $\operatorname{Ext}_{B \rightarrow D}^{\Gamma}(Q, B, \psi)$. The correspondence $[F, \widetilde{F}] \mapsto\left[\mathcal{E}_{F}\right]$ defines an injection

$$
\Omega: \operatorname{Hom}_{(\psi, 0)}\left[\operatorname{Dis}_{\Gamma} Q, \mathbb{P}_{B \rightarrow D}\right] \rightarrow \operatorname{Ext}_{B \rightarrow D}^{\Gamma}(Q, B, \psi)
$$

thanks to Step 1 below.

Step 1: The $\Gamma$-graded monoidal functors $F, F^{\prime}:{ }_{\Gamma}$ Dis $Q \rightarrow \mathbb{P}$ are homotopic if and only if the corresponding associated equivariant extensions $\mathcal{E}_{F}, \mathcal{E}_{F^{\prime}}$ are equivalent.

We first recall that every graded monoidal functor $(F, \widetilde{F})$ is homotopic to one $(G, \widetilde{G})$ in which $G(1)=1$. Hence, we can restrict our attention to this kind of graded monoidal functors.

Let $F, F^{\prime}:{ }_{\Gamma}$ Dis $Q \rightarrow \mathbb{P}$ be homotopic by a homotopy $\alpha: F \rightarrow F^{\prime}$. Then, there exists a function $g: Q \rightarrow B$ such that $\alpha_{x}=(g(x), 1)$, that is,

$$
F x=d g(x) F^{\prime} x \text {. }
$$


The naturality of $\alpha$ gives

$$
f(x, \sigma)+g(\sigma x)=\sigma g(x)+f^{\prime}(x, \sigma) .
$$

The coherence condition (1) of the homotopy $\alpha$ implies $g(1)=0$ and

$$
f(x, y)+g(x y)=g(x)+\vartheta_{F^{\prime} x} g(y)+f^{\prime}(x, y) .
$$

Let $\mathcal{E}_{F^{\prime}}$ be the extension associated to $F^{\prime}$ by Lemma 9 . We write

$$
\begin{aligned}
\alpha^{*}: E_{F} & \rightarrow E_{F^{\prime}} \\
(b, x) & \mapsto(b+g(x), x)
\end{aligned}
$$

Then, thanks to the equations (15) and (16), $\alpha^{*}$ is a $\Gamma$-homomorphism. Further, the following diagram commutes
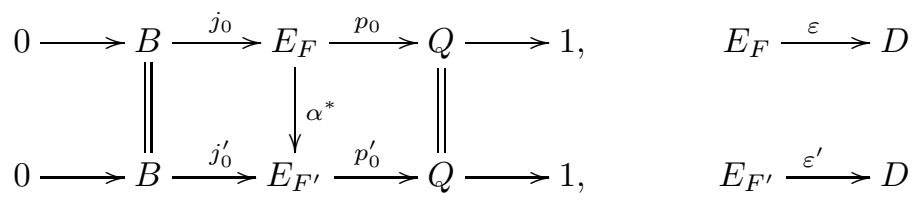

and hence $\alpha^{*}$ is an isomorphism. It remains to show that $\varepsilon^{\prime} \alpha^{*}=\varepsilon$. It follows from the equations (13) and (14) that

$$
\begin{aligned}
\varepsilon^{\prime} \alpha^{*}(b, x) & =\varepsilon^{\prime}(b+g(x), x)=d(b+g(x)) F^{\prime} x \\
& =d(b) d(g(x)) F^{\prime} x=d(b) F x=\varepsilon(b, x) .
\end{aligned}
$$

Thus, two extensions $\mathcal{E}_{F}$ and $\mathcal{E}_{F^{\prime}}$ are equivalent.

Conversely, if $\alpha^{*}: E_{F} \rightarrow E_{F^{\prime}}$ is an isomorphism, then

$$
\alpha^{*}(b, x)=(b+g(x), x)
$$

where $g: Q \rightarrow B$ is a function with $g(1)=0$. Thus, $\alpha_{x}=(g(x), 1)$ is a homotopy of $F$ and $F^{\prime}$ as we see by retracing our steps.

Step 2: $\Omega$ is surjective.

Assume that $\mathcal{E}$ is an equivariant extension of $B$ by $Q$ of type $B \rightarrow D$ inducing $\psi: Q \rightarrow$ Coker $d$ as in the commutative diagram (9). We prove that $\mathcal{E}$ is equivalent to an extension $\mathcal{E}_{F}$ associated to some $\Gamma$-graded monoidal functor $(F, \widetilde{F}):{ }_{\Gamma} \operatorname{Dis} Q \rightarrow \mathbb{P}_{B \rightarrow D}$.

For each $x \in Q$, choose a representative $u_{x} \in E$ such that $p\left(u_{x}\right)=x, u_{1}=0$. An element in $E$ can be uniquely written as $b+u_{x}$ for $b \in B, x \in Q$. The representatives $\left\{u_{x}\right\}$ induce a normalized function $f: Q \times Q \cup Q \times \Gamma \rightarrow B$ by

$$
\begin{gathered}
u_{x}+u_{y}=f(x, y)+u_{x y}, \\
\sigma u_{x}=f(x, \sigma)+u_{\sigma x},
\end{gathered}
$$

and the automorphisms $\varphi_{x}$ of $B$ by

$$
\varphi_{x}=\mu_{u_{x}}: b \mapsto u_{x}+b-u_{x} .
$$


It follows from the condition $H_{2}$ of the homomorphism $(i d, \varepsilon)$ of $\Gamma$-crossed modules that

$$
\vartheta_{\varepsilon u_{x}}=\mu_{u_{x}}=\varphi_{x} .
$$

Then, the $\Gamma$-group structure of $E$ can be described by

$$
\begin{gathered}
\left(b+u_{x}\right)+\left(c+u_{y}\right)=b+\varphi_{x}(c)+f(x, y)+u_{x y}, \\
\sigma\left(b+u_{x}\right)=\sigma b+f(x, \sigma)+u_{\sigma x} .
\end{gathered}
$$

Since $\psi(x)=\psi p\left(u_{x}\right)=q \varepsilon\left(u_{x}\right), \varepsilon\left(u_{x}\right)$ is a representative of $\psi(x)$ in $D$. Thus, we define a $\Gamma$-graded monoidal functor $(F, \widetilde{F}): \operatorname{Dis}_{\Gamma} Q \rightarrow \mathbb{P}$ as follows.

$$
F x=\varepsilon\left(u_{x}\right), F(x \stackrel{\sigma}{\rightarrow} \sigma x)=(f(x, \sigma), \sigma), \widetilde{F}_{x, y}=(f(x, y), 1) .
$$

The equations (18) and (17) show that $F(\sigma)$ and $\widetilde{F}_{x, y}$ are actually morphisms in $\mathbb{P}$, respectively. The normality of the function $f(x, \sigma)$ gives $F\left(i d_{x}\right)=i d_{F x}$. Clearly, $F(1)=1$. This together with the normality of the function $f(x, y)$ imply the compatibility of $(F, \widetilde{F})$ with the unit constraints. The associativity law and the $\Gamma$-group properties of $B$ imply the equations (6)-(8), respectively, in which $\vartheta_{F x}$ is replaced by $\varphi_{x}$. These equations show that $(F, \widetilde{F})$ is compatible with the associativity constraints, $\widetilde{F}_{x, y}$ is a natural isomorphism and $F$ preserves the composition of morphisms, respectively.

Finally, it is easy to check that the equivariant crossed product extension $\mathcal{E}_{F}$ associated to $(F, \widetilde{F})$ is equivalent to the extension $\mathcal{E}$ by the $\Gamma$-isomorphism $\alpha:(b, x) \mapsto b+u_{x}$.

Moreover, each equivariant group extension of $B$ by $Q$ studied in [6] may be viewed as an equivariant group extension of type of $\Gamma$-crossed module $(B$, Aut $B, \mu, 0)$. Then, $\mathbb{P}_{B \rightarrow \text { Aut } B}$ is just the holomorph $\Gamma$-graded categorical group of a $\Gamma$-group $B, \mathrm{Hol}_{\Gamma} B$.

Corollary 11 ([6, Theorem 4.2]). For $\Gamma$-groups $B$ and $Q$, there exists a bijection

$$
\operatorname{Ext}_{\Gamma}(Q, B) \leftrightarrow \operatorname{Hom}_{\Gamma}\left[\operatorname{Dis}_{\Gamma} Q, \operatorname{Hol}_{\Gamma} B\right] .
$$

Let $\mathbb{P}=\mathbb{P}_{B \rightarrow D}$ be the $\Gamma$-graded categorical group associated to the $\Gamma$-crossed module $B \rightarrow D$. Since $\pi_{0} \mathbb{P}=$ Coker $d$ and $\pi_{1} \mathbb{P}=$ Ker $d$, the reduced graded categorical group of $\mathbb{P}$ is

$$
\mathcal{S}_{\mathbb{P}}=\int_{\Gamma}(\text { Cokerd, Kerd }, h), h \in Z_{\Gamma}^{3}(\text { Cokerd }, \text { Kerd }) .
$$

Then, by (2), the pair of $\Gamma$-homomorphisms $(\psi, 0):(Q, 0) \rightarrow($ Cokerd, Kerd $)$ induces an obstruction

$$
\psi^{*} h \in Z_{\Gamma}^{3}(Q, \operatorname{Ker} d) .
$$

Under this notion of obstruction, we state the following theorem. 
Theorem 12. Let $(B, D, d, \vartheta)$ be a $\Gamma$-crossed module, and let $\psi: Q \rightarrow$ Cokerd be a $\Gamma$-homomorphism. Then, the vanishing of $\overline{\psi^{*} h}$ in $H_{\Gamma}^{3}(Q, \operatorname{Kerd})$ is necessary and sufficient for there to exist an equivariant extension of $B$ by $Q$ of type $B \rightarrow D$ inducing $\psi$. Further, if $\overline{\psi^{*} h}$ vanishes, then the equivalence classes of such extensions are bijective with $H_{\Gamma}^{2}(Q, \operatorname{Kerd})$.

Proof. By the assumption, $\overline{\psi^{*} h}=0$, thus by Proposition 2, there exists a $\Gamma$ graded monoidal functor $(\Psi, \widetilde{\Psi}):{ }_{\Gamma} \operatorname{Dis} Q \rightarrow \mathcal{S}_{\mathbb{P}}$. Then, composition of $(\Psi, \widetilde{\Psi})$ and $(H, \widetilde{H}): \mathcal{S}_{\mathbb{P}} \rightarrow \mathbb{P}$ is a $\Gamma$-graded monoidal functor $(F, \widetilde{F}):{ }_{\Gamma} \operatorname{Dis} Q \rightarrow \mathbb{P}$. It is easy to see that $F$ induces the pair of $\Gamma$-homomorphisms $(\psi, 0)$, hence by Lemma 9, we obtain an associated extension $\mathcal{E}_{F}$.

Conversely, suppose that there is an equivariant extension as in the diagram (9). Let $\mathbb{P}^{\prime}$ be the $\Gamma$-graded categorical group associated to the $\Gamma$ crossed module $B \rightarrow E$. By Lemma 5 , there is a $\Gamma$-graded monoidal functor $F: \mathbb{P}^{\prime} \rightarrow \mathbb{P}$. Since the reduced graded categorical group of $\mathbb{P}^{\prime}$ is ${ }_{\Gamma}$ Dis $Q$, by Proposition $1, F$ induces a $\Gamma$-graded monoidal functor of type $(\psi, 0)$ from ${ }_{\Gamma}$ Dis $Q$ to $\mathcal{S}_{\mathbb{P}}=\int_{\Gamma}(\operatorname{Coker} d, \operatorname{Ker} d, h)$. Now, by Proposition 2 , the obstruction of the pair $(\psi, 0)$ vanishes in $H_{\Gamma}^{3}(Q, \operatorname{Ker} d)$, that is, $\overline{\psi^{*} h}=0$.

The final assertion of the theorem follows from Proposition 2 and Theorem 10 .

Note that if $\Gamma=\mathbf{1}$, the trivial group, then the set $\operatorname{Ext}_{B \rightarrow D}^{\mathbf{1}}(Q, B, \psi)$ is just the set of equivalence classes of group extensions of the type of a crossed module studied in $[2,8,16]$. Thus, we obtain the following consequence.

Corollary 13 ([2, Theorem 5.2]). Let $(B, D, d, \vartheta)$ be a crossed module, and let $\psi: Q \rightarrow$ Cokerd be a group homomorphism. Then, there exists a 3-dimensional cohomology class $k(B, D, \psi) \in H^{3}(Q, \mathrm{Kerd})$, called the obstruction, whose vanishing is necessary and sufficient for there to exist an extension of $B$ by $Q$ of type $B \rightarrow D$ inducing $\psi$. Further, if $k(B, D, \psi)$ vanishes, then the equivalence classes of such extensions are bijective with $H^{2}(Q, \operatorname{Kerd})$.

For the $\Gamma$-crossed module $(B$, Aut $B, \mu, 0)$, since Coker $\mu=\operatorname{Out} B$, Ker $\mu=$ $Z(B)$, Theorem 12 contains Theorem 4.1 [6].

Corollary 14 ([6, Theorem 4.1]). Let B, D be $\Gamma$-groups and let $\psi: Q \rightarrow$ Out $B$ be a $\Gamma$-homomorphism. Then, there exists the obstruction $\operatorname{Obs}(\psi) \in$ $H_{\Gamma}^{3}(Q, Z(B))$ whose vanishing is necessary and sufficient for there to exist an equivariant extension of $B$ by $Q$ inducing $\psi$. Further, if $\operatorname{Obs}(\psi)$ vanishes, then the equivalence classes of such extensions are bijective with $H_{\Gamma}^{2}(Q, Z(B))$.

\section{References}

[1] J. C. Baez and A. D. Lauda, Higher dimensional algebra V: 2-groups, Theory Appl. Categ. 12 (2004), 423-491.

[2] R. Brown and O. Mucuk, Covering groups of non-connected topological groups revisited, Math. Proc. Cambridge Philos. Soc. 115 (1994), no. 1, 97-110. 
[3] R. Brown and C. B. Spencer, $\mathcal{G}$-groupoids, crossed modules and the fundamental groupoid of a topological group, Indag. Math. 38 (1976), no. 4, 296-302.

[4] M. Calvo, A. M. Cegarra, and N. T. Quang, Higher cohomologies of modules, Algebr. Geom. Topol. 12 (2012), no. 1, 343-413.

[5] A. M. Cegarra, A. R. Garzón, and J. A. Ortega, Graded extensions of monoidal categories, J. Algebra 241 (2001), no. 2, 620-657.

[6] A. M. Cegarra, J. M. García-Calcines, and J. A. Ortega, On graded categorical groups and equivariant group extensions, Canad. J. Math. 54 (2002), no. 5, 970-997.

[7] Cohomology of groups with operators, Homology Homotopy Appl. 4 (2002), no. $1,1-23$.

[8] P. Dedecker, Les foncteurs $E x t_{\Pi}, H_{\Pi}^{2}$ et $H_{\Pi}^{2}$ non abéliens, C. R. Acad. Sci. Paris 258 (1964), 4891-4894.

[9] A. Fröhlich and C. T. C. Wall, Graded monoidal categories, Compos. Math. 28 (1974), 229-285.

[10] A. Grothendieck, Catégories fibrées et déscente, (SGA I, exposé VI), Lecture Notes in Math. 224, 145-194, Springer, Berlin, 1971.

[11] A. Joyal and R. Street, Braided tensor categories, Adv. Math. 82 (1993), no. 1, 20-78.

[12] B. Noohi, Group cohomology with coefficients in a crossed-module, J. Inst. Math. Jussieu 10 (2011), no. 2, 359-404.

[13] N. T. Quang and P. T. Cuc, Crossed bimodules over rings and Shukla cohomology, Math. Commun. 17 (2012), no. 2, 575-598.

[14] N. T. Quang, P. T. Cuc, and N. T. Thuy, Crossed modules and strict Gr-categories, Comm. Korean Math. Soc. 29 (2014), no. 1, 9-22.

[15] N. T. Quang, N. T. Thuy, and P. T. Cuc, Monoidal functors between (braided) Grcategories and their applications, East-West J. Math. 13 (2011), no. 2, 163-186.

[16] R. L. Taylor, Compound group extensions I, Trans. Amer. Math. Soc. 75 (1953), 106135.

[17] K.-H. Ulbrich, Group cohomology for Picard Categories, J. Algebra 91 (1984), no. 2, 464-498.

[18] - On cohomology of graded group categories, Compos. Math. Tome 63 (1987), no. 3, 409-417.

[19] J. H. C. Whitehead, Combinatorial homotopy II, Bull. Amer. Math. Soc. 55 (1949), $453-496$.

Pham Thi CuC

Natural Science Department

HongduC UNIVERSITY

ThanhHoA, Vietnam

E-mail address: cucphamhd@gmail.com

NGUYen TiEn QuANG

Department of Mathematics

HANOI NATIONAL UNIVERSity OF EDUCATION

HANOI, ViETNAM

E-mail address: cn.nguyenquang@gmail.com 